\title{
Corrections
}

\section{Correction: Qi et al., Coordinated Regulation of Dendrite Aborization by Epigenetic Factors CDYL and EZH2}

In the article "Coordinated Regulation of Dendrite Aborization by Epigenetic Factors CDYL and EZH2" by Cai Qi, Shumeng Liu, Rui Qin, Yu Zhang, Guoqiang Wang, Yongfeng Shang, Yun Wang, and Jing Liang, which appeared on pages 4494-4508 of the March 26, 2014 issue, the wrong sequences for nonsilencing shRNA gene appeared in the Materials and Methods on page 4495, left column, lines 30-33, as follows: sense: 5' -GATCCCC-GCAAATTCTCGGTGTCAAA-TTCAAGAGA-TTTGACACCGAGAATTTGC-TTTTTA-3'; antisense: 5'-AGCTTAAAAA-GCAAATTCTCGGTGTCAAA-TCTCTTGAA-TTTGACACCGAGAATTTGC-GGG-3'. The authors used the correct nonsilencing shRNA 5' -GATCCCC-TTCTCCGAACGTGTCACGT-TTCAAGAGA-ACGTGACACGTTCGGAGAATTTTTA-3'; antisense: 5' -AGCTTAAAAA-TTCTCCGAACGTGTCACGT-TCTCTTGAA-ACGTGACACGTTCGGAGAA-GGG-3' during all the experiments. This correction does not affect any results or interpretation of the data.

DOI:10.1523/JNEUROSCI.5266-14.2015

\section{Correction: Kummer et al., Ear2 Deletion Causes Early Memory and Learning Deficits in APP/PS1 Mice}

In the article "Ear2 Deletion Causes Early Memory and Learning Deficits in APP/PS1 Mice" by Markus P. Kummer, Thea Hammerschmidt, Ana Martinez, Dick Terwel, Gregor Eichele, Anika Witten, Stefanie Figura, Monika Stoll, Stephanie Schwartz, Hans-Christian Pape, Joachim L. Schultze, David Weinshenker, and Michael T. Heneka, which appeared on pages 8845-8854 of the June 25, 2014 issue, an oversight on the part of some co-authors led to the omission of one author whose contributions the corresponding author was unaware of. The author list should include Inga Urban, Max Planck Institute of Biophysical Chemistry, who performed the respective HPLC measurements and analyzed the data set. The corrected author list is as follows: "Markus P. Kummer, Thea Hammerschmidt, Ana Martinez, Dick Terwel, Gregor Eichele, Anika Witten, Stefanie Figura, Monika Stoll, Stephanie Schwartz, Inga Urban, HansChristian Pape, Joachim L. Schultze, David Weinshenker, and Michael T. Heneka." The Author Contributions footnote should have read “Author contributions: M.P.K., T.H., D.T., G.E., H.-C.P., J.L.S., D.W., and M.T.H. designed research; M.P.K., T.H., A.M., D.T., A.W., S.F., M.S., and S.S. performed research; M.P.K., T.H., A.M., D.T., A.W., S.F., M.S., and I.U. analyzed data; M.P.K., T.H., I.U., D.W., and M.T.H. wrote the paper.

DOI:10.1523/JNEUROSCI.5263-14.2015 